\title{
Una aproximación a la teoría del Public Choice y sus implicancias en los mecanismos de control estatal
}

\section{Christian Guzmán Napurí}

\section{Introducción}

En el año de 1991, un abogado obtuvo el Premio Nobel de Economía por haber descubierto lo que a primera vista parece una perogrullada: el contratar cuesta. El celebrar contratos genera costos en dinero, tiempo y esfuerzo que en diversas circunstancias puede hacer difíciles y hasta imposibles las transacciones en el mercado. A ello Ronald Coase llamó costos de transacción.

Asimismo, James Buchanan, un economista de la Universidad de Virginia, descubrió que era posible la aplicación y la extensión de la teoría económica a las opciones políticas y gubernamentales y además, encontró que también era posible extender dicha teoría a las decisiones de los ciudadanos para efectos de hacer posible la elección entre las diversas opciones existentes'.

Asimismo, y como resultado del análisis anterior, Buchanan realizó un descubrimiento que también parece una verdad de perogrullo: Las decisiones políticas, que son las realizadas por los estamentos del Estado encargados del manejo estatal, necesariamente generan costos a los particulares y a la sociedad en su conjunto. Asimismo, las decisiones políticas no

1 La posibilidad de aplicación del análisis económico a hechos distintos a los meramente patrimoniales no resulta ser novedad. Ya otro Premio Nobel de Economía, Gary Becker, había señalado la posibilidad de aplicar el método de estudio de la economía para entender prácticamente cualquier. Gary Becker, «El Enfoque Económico del Comportamiento Humano". Introducción de libro The Economic Approach to Human Behaviour. University of Chicago Press, 1976. 
le cuestan al Estado como tal, sino a todos los que se encuentran bajo el dominio de dicho estado ${ }^{2}$.

Como resultado de la constatación referida en los párrafos precedentes, y dado que los Estados administran bienes y fondos, los cuales por definición son públicos, el pueblo debería tener la posibilidad de recurrir a mecanismos previstos por la ley y de obligatorio cumplimiento, que le permitan controlar e intervenir en las decisiones de los actores públicos.

Todo el razonamiento antes descrito configura, a muy grandes rasgos, parte importante de los conceptos a través de los cuales Buchanan también obtiene el Premio Nobel de Economía, y que configuran la llamada Teoría de la Elección Pública (Public Choice). La teoría antes referida resulta importante para comprender diversos fenómenos políticos y jurídicos que se dan en la realidad. Asimismo, permite entender la razón por la cual las normas jurídicas constitucionales deben elaborarse de tal forma que permitan un adecuado control del comportamiento del Estado por parte del electorado y además permite entender también porque dicho control resulta indispensable.

En este orden de ideas, son las Constituciones de los Estados las encargadas de hacer posible dicho control. Ello, que también parece una verdad evidente, no se ha tenido en cuenta en la mayoría de los países en los cuales existe una norma constitucional más o menos coherente. Y es que en realidad lejos de configurar - y/o de regular adecuadamente - mecanismos de fiscalización entre electores y gobiernos, las constituciones políticas de las diversas naciones elaboran únicamente controles al interior del Estado, amparados en el concepto de separación de poderes ${ }^{3}$, concepto que resulta ser un presupuesto necesario, pero que no resulta ser suficiente para conjurar la posibilidad de un régimen autoritario.

2 El artículo de Buchanan que expresa claramente esta teoría es: James Buchanan, "From Private Preferences to Public Philosophy: The Development of de Public Choice». Trabajo publicado en el volumen: The Economics of Politics, IEA, Londres, 1978, pp. 1-20.

3 El Baron de Montesquieu, quien explicita por primera vez el principio de separación de poderes señalaba que el Estado poseía tres clases de poderes - legislativo, ejecutivo y judicial - y que no podía existir libertad política si más de uno de dichos poderes residían "en la misma persona o el mismo cuerpo». Partía Montesquieu del supuesto de que el poder podría controlar al poder. Baron de Montesquieu, Del Espíritu de las Leyes. Buenos Aires, Ediciones Orbis S.A., 1984, p. 141 y ss. En general, Montesquieu trata el tema en todo el Libro XI de su tratado.

Debemos señalar además que el principio ha sufrido modificaciones, en especial en el ámbito europeo. Las funciones del Estado están compartidas entre los diversos órganos del 
Y es que, una vez aplicado el análisis económico al comportamiento del funcionario público ${ }^{4}$, debemos concluir necesariamente que éste, como ser racional, busca maximizar la utilidad de su cargo, es decir, obtener el máximo provecho del mismo. Y, como resultado de ello cuando el funcionario es un político con un cargo importante, éste va a buscar crear las condiciones adecuadas para poder ser reelegido, así como procurar elevar la cuota de poder de la que goza. Como evidente consecuencia de ello, debemos deducir que el Estado tiende necesariamente a elevar el poder material del que goza. Resulta entonces una utopía considerar que el Estado, al fin, un ente monolítico, podrá controlarse a sí mismo, no obstante que existan en su interior mecanismos de fiscalización entre órganos.

Los razonamientos contenidos en los párrafos que anteceden han sido desconocidos sistemáticamente por gran parte de los ordenamientos jurídicos del Mundo, que siguen suponiendo sin sustento alguno que el funcionario público es un ser económicamente neutro y que obra siempre en términos de «interés común» ${ }^{5}$. Semejante consideración es totalmente inconsistente, pero no solamente con la teoría expuesta, sino también con lo observado empíricamente en las sociedades modernas.

Es así como el control al que estamos haciendo referencia no se encuentra enfocado únicamente en el Estado como tal, sino además en las personas que conforman dicha entidad, seres humanos comunes y corrientes que no tienen mayor diferencia con el elector común que el desempeño de un cargo público. Esta es la razón por la cual las constituciones deben contener normas que permitan conciliar el interés del funcionario con el interés común, expresado en términos de eficiencia social. Es decir, como en el mercado patrimonialmente considerado, debe propenderse a que el comportamiento individual favorezca los intereses del grupo. Las normas constitucionales y las normas legales relacionadas

mismo, órganos que deben controlarse mutuamente. De ahí parten principios como el de la interdependencia entre órganos del Estado. Al respecto: Karl Loewenstein, Teoría de la Constitución. Barcelona, Editorial Ariel, 1976, p. 54 y ss.

4 Cuando hablemos de funcionario público, para los efectos del presente trabajo, nos referiremos necesariamente a toda persona que labore para el Estado, sin importar la función específica que desempeña.

5 Ver al respecto: James Buchanan, Op. cit. p. 18-19. Gordon Tullock, "El Voto como un Medio de Control Colectivo". En: Gordon Tullock, Los Motivos del Voto, Editorial Espasa Calpe S.A., Madrid 1979, Cap. III, p. 39. En la misma línea: Folke Kafka, Teoría Económica. Lima, Universidad del Pacífico, 1981, p. 612 y ss. 
con el funcionamiento del Estado tienen el especial encargo de hacer posible la referida canalización.

\section{Mecanismos de control elector-estado en las constituciones modernas}

Como lo hemos expresado líneas arriba, el control entre electores y el Estado se justifica plenamente dada la constatación, en la teoría y empíricamente, que no basta con los controles al interior del estado para evitar que éste degenere en el llamado "Estado Leviatán" ${ }^{6}$. Dado que los gobiernos se encuentran conformados por personas que buscan maximizar su utilidad, estos procurarán, ante la ausencia de controles efectivos, elevar su cuota de poder. En este orden de ideas, debemos señalar en un primer término que tales controles son posibles únicamente en una sociedad democrática, ya que tanto desde el punto de vista jurídico, como desde una perspectiva fáctica, una dictadura no puede ser controlada por el electorado en forma alguna.

Asimismo, el hecho de que aquellos que conforman el gobierno deben ser elegidos mediante el sufragio universal permite hacer posible cierto control. En este punto, es preciso señalar que si bien es cierto que la elección de las autoridades políticas mediante el voto directo configura un elemento importante de participación política, la posibilidad de participar no debe agotarse en dicha elección, sino más bien es necesaria la existencia de mecanismos establecidos por la constitución destinados a hacer posible un control más o menos inmediato de las decisiones políticas por parte del electorado.

6 Algunos analistas han acuñado esta frase para referirse al desmedido crecimien to de la administración pública norteamericana, crecimiento que no tiene control alguno que no provenga del propio Estado. Si bien esto es cierto, también es cierto que el Estado norteamericano no degenera en el autoritarismo dada la existencia de un eficiente mecanismo de checks and balances (frenos y contrapesos).

Sin embargo, justo es reconocer que todo lo expuesto hace que los costos de mantenimiento del sistema resulten ser muy altos. El temor de ciertos analistas entonces estriba en la posibilidad de un colapso, generado en especial por problemas presupuestarios derivados de la falta de equilibrio financiero. James Buchanan, Op. cit. p. 18. 


\section{Control de la Autoridad Política}

En primer término, y dado lo expuesto en las líneas precedentes, es necesario que la norma constitucional de cada nación preceptúe mecanismos que permitan que el electorado tenga participación en el manejo del Estado, manejo cuya responsabilidad reside directamente en aquellos funcionarios que fueron elegidos por el pueblo.

Como lo hemos señalado en un trabajo anterior ${ }^{7}$, la democracia representativa encuentra su justificación en el hecho que las personas que fueron elegidas deban, al tomar una decisión política, «simular» la determinación a la que habrían llegado los particulares de haberse podido poner de acuerdo. En consecuencia, las normas legales que organizan el Estado, que generalmente parten de la Norma Constitucional de los mismos, pretenden que las decisiones estatales se tomen de forma tal que asemejen lo más posible la determinación a la que hemos hecho referencia ${ }^{8}$.

No obstante lo anteriormente expuesto, por diversas circunstancias, entre las cuales sin duda encontramos las de un inadecuado diseño constitucional o la de la ausencia de una tradición democrática (situación desafortunadamente muy frecuente en países como los nuestros), la elección mediante sufragio no basta en forma alguna para que la simulación antes señalada se cumpla a cabalidad y la decisión de la autoridad política no se asemeja siquiera a la postura que en su caso asumiría el electorado. Por ello, resulta indispensable el establecimiento de mecanismos que permitan corregir esa situación, permitiendo así que el electorado pueda controlar directa o indirectamente, por lo menos parte de la decisión política que se tome.

\subsection{Recurrencia a mecanismos de democracia participativa}

Por lo anteriormente expuesto, los particulares deben tener la posibilidad de utilizar los ahora denominados mecanismos de democracia participativa, los mismos que tienen por finalidad permitir al elector intervenir en las decisiones públicas. Desde el punto de vista del Análisis

7 En un trabajo anterior, hemos demostrado, a través de consideraciones económicas, que la democracia es el sistema político más coherente. Christian Guzmán Napurí, „Una aproximación a la aplicación del análisis económico del derecho al derecho constitucional». En: Revista Derecho y Sociedad, No 15, Lima, PUCP, 2000.

8 Christian Guzmán Napurí, Op. cit. 
Económico del Derecho, en muchas ocasiones resulta más eficiente trasladar la decisión política al electorado que dejar que esta sea tomada por la autoridad política, por más que ésta haya sido elegida por el pueblo. Ello ocurre cuando el costo en términos de gobernabilidad que tendría que asumir el Estado, si es que toma una decisión que no encuentra un correlato adecuado en las preferencias del electorado, podría ser tan alto que generaría una situación de inestabilidad tal que incluso pondría en peligro el propio régimen imperante.

Debemos señalar también que la existencia de mecanismos de participación política permite acercarnos a modelos de democracia más cercanos a la llamada democracia deliberativa ${ }^{9}$, concepto derivado de los trabajos de Jurgen Habermas. La democracia deliberativa, a diferencia de la democracia representativa clásica, pretende eliminar, o por lo menos menguar, la desigualdad formal y material existente entre gobernantes y gobernados, a través fundamentalmente de los mecanismos de comunicación y diálogo ${ }^{10}$. Sin embargo, la igualdad como la antes señalada es imposible en términos reales. Un esquema intermedio es precisamente el de la democracia participativa o semidirecta, en la cual se mantienen las desigualdades antedichas, pero estas se encuentran mediatizadas a través de los mecanismos de participación política de la ciudadanía.

Los razonamientos expuestos en los párrafos precedentes, desde el punto de vista doctrinario y en especial, a la luz de los conceptos provenientes del análisis económico, nos permiten justificar la existencia de ciertos mecanismos de participación política como podrían ser, entre otros, la iniciativa legislativa, el referéndum, y la revocatoria de autoridades.

En general, los mecanismos de participación política a los que hacemos referencia requieren la necesidad de la participación del electorado por propia iniciativa a fin de activar el proceso. Ello, si es que el citado mecanismo no es activado por el propio Estado en supuestos muy especiales establecidos por el ordenamiento jurídico. En el caso particular del

9 Eduardo Hernando Nieto, "Teorías Democráticas hoy: ¿Cuál de ellas garantiza la igualdad, la libertad y la virtud?». En: Revista de Derecho PUCP; Lima, PUCP, 1998 1999, p. 328 y ss.

10 Es interesante señalar que Habermas critica la racionalidad instrumental propia de la ilustración y pretende reemplazarla por el concepto de racionalidad comunicativa, la misma que llevaría a las naciones a un verdadero progreso. Sin embargo, la racionalidad comunicativa también partía del presupuesto del ser humano como ente racional, en el sentido económico del término. 
referéndum y la revocatoria de autoridades, ambas instituciones tienen en común ciertos elementos en su diseño, destacando en primer lugar la necesidad de la convocatoria al proceso determinado, realizada por el propio Estado, a iniciativa de éste o de cierto número de particulares a los que la Ley les asigna la legitimidad para activar el proceso. Por otro lado, la decisión o el acuerdo se toma a partir del voto directo y personal, esquema que permite reducir los costos en los que incurriría el elector para la toma de decisiones.

\section{La Iniciativa Legislativa}

En particular, debemos señalar que la llamada iniciativa legislativa es un mecanismo que permite a los particulares la participación en la elaboración legislativa realizada por el Parlamento. La norma constitucional permitiría a los electores, cumpliendo con ciertos requisitos establecidos por la ley, la presentación de proyecros de ley, los mismos que podrán ser discutidos por el Parlamento y luego, siguiendo el trámite establecido para la aprobación de las leyes, podrían convertirse en una norma legal.

Es preciso indicar que, en muchos casos, en el derecho nacional y comparado, la iniciativa legislativa ha sido facultad reservada únicamente a los órganos del gobierno y en ciertos casos, únicamente a los miembros del Parlamento. Es relativamente reciente su ampliación a supuestos de intervención del electorado.

Por otro lado, la iniciativa legislativa, como mecanismo de participación, adolece de ciertas limitaciones. En primer lugar, el elector común no goza de las facilidades para el acceso a la información de las que gozaría un funcionario público. Dicha información resulta indispensable a fin de tomar decisiones adecuadas ${ }^{11}$. Por otro lado, la iniciativa legislativa es susceptible de ser manipulada por intereses puntuales de grupos económicos o sociales determinados. Finalmente, no existe la posibilidad de un control directo del proceso una vez iniciado éste. El electorado, una vez que ha presentado el proyecto de Ley, no tendría intervención en su aprobación o promulgación.

11 Peter Dienel y Hans Harms, Repensar la Democracia. Barcelona, Ediciones del Serbal, 2000, p. 55. 


\section{La Consulta Popular}

La consulta popular, o referéndum, es el típico mecanismo de democracia participativa, el instrumento que permitiría trasladar del Estado al electorado una disputa o la determinación respecto de una decisión política. El referéndum permite al elector poder participar en la toma de decisiones que de otra forma resultaría imposible siquiera controlar. En este orden de ideas es necesario diferenciar el concepto de referéndum del de plebiscito. El primero resulta ser una consulta respecto a la validez o vigencia de una norma legal. El plebiscito es, en cambio, una consulta sobre cuestiones más variadas, que pueden implicar incluso la legitimidad del gobierno de turno.

En este orden de ideas, no siempre se han escuchado, en la doctrina constitucional moderna, voces favorables al establecimiento de las instituciones analizadas en el párrafo precedente, en especial en lo que concierne al plebiscito. Un primer lugar, el procedimiento es susceptible de ser afectado por influencias ajenas e intervención política ${ }^{12}$. Como resultado, las instituciones materia de análisis se han utilizado muchas veces como un mecanismo para dar validez a gobiernos autoritarios, dada la facilidad de la que podrían gozar éstos últimos para manipular a la opinión pública.

Por otro lado, al igual que en el caso de la iniciativa legislativa, el elector común no goza de las facilidades para el acceso a la información de las que gozaría un funcionario público. Al igual que en el mercado patrimonialmente considerado, en el mercado político - concepto al cual hemos hecho y haremos referencia reiteradamente en el presente trabajo se requiere que el consumidor maneje información adecuada respecto de los bienes o servicios respecto de los cuales va a contratar.

Finalmente, y dada la dificultad material de que los electores puedan ponerse de acuerdo respecto a cuestiones complejas, la consulta popular sirve para discutir temas muy puntuales, de características determinadas ${ }^{13}$. Normalmente, la decisión del electorado se centra en respuestas del tipo si/no respecto a uno o varios temas, lo cual es a todas luces limitante. 


\section{La revocatoria de autoridades}

Por otro lado, una institución como la revocatoria de autoridades adecuadamente diseñada, claro está - podría facilitar la corrección de situaciones en las cuales los miembros del gobierno pierden representación. La revocatoria de autoridades permite que los particulares, cumpliendo ciertos requisitos establecidos en la Ley, puedan poner fin al mandato de una autoridad pública elegida mediante el voto popular directo.

Es necesario precisar que, en principio, en prácticamente ninguna constitución del planeta existe mecanismo alguno de participación del electorado que permita revocar en forma directa el mandato de algún miembro del Gobierno Central. La posibilidad de revocatoria se restringe a las relaciones entre órganos, en especial entre Gobierno y Parlamento. Además, en el derecho comparado, la revocatoria de autoridades está diseñada, en principio, únicamente en función a gobiernos regionales y locales ${ }^{14}$. Este es el caso en el cual resultaría sumamente interesante el crear mecanismos que permitieran activar la revocatoria de autoridades a fin de afectar políticamente, por ejemplo, a los miembros del Parlamento.

Finalmente y como explicaremos más adelante, la pérdida de representatividad del Gobierno de turno echa por tierra la justificación de la existencia de la democracia representativa, que es la necesidad de simular la decisión del electorado en un momento político dado. En un escenario como el descrito, un mecanismo como la revocatoria de autoridades permitiría recomponer la conformación de las instituciones políticas que hayan perdido representatividad.

\subsection{La recurrencia a mecanismos de arbitraje electoral}

De la misma forma como deben existir mecanismos de Democracia Participativa amparados constitucionalmente, mecanismos a los que hemos hecho referencia líneas arriba, también existe la necesidad de que una constitución prevea mecanismos a través de los

14 El caso más cercano es el de la propia Constitución Peruana del año 1993, la misma que restringe el derecho de revocatoria de autoridades a los gobiernos locales y regionales, no siendo posible siquiera revocar el mandato de congresistas. A mayor abundamiento, resulta impensable, por lo menos a la luz del derecho comparado, la existencia de un derecho de revocatoria del mandato presidencial, máxime si el régimen es presidencialista. Cuando el Jefe de Estado no llega al cargo mediante el voto popular (es el caso de las monarquías europeas, por ejemplo) la posibilidad de revocatoria es evidentemente inviable. 
cuales alguno de los estamentos del Estado deba recurrir al electorado a fin que éste actúe como árbitro ante situaciones que el propio Estado no pueda corregir eficientemente en su seno. El típico mecanismo para ello es el de la llamada Disolución Parlamentaria, que consiste en la revocatoria del mandato de los miembros del Parlamento por parte, en general, del Jefe de Estado. La institución reseñada existe en prácticamente todas las naciones que poseen un régimen parlamentario de gobierno, pero se encuentra ausente en la mayoría de constituciones de naciones de régimen presidencial, sea este puro o atenuado15.

La disolución parlamentaria resulta fundamental en escenarios en los cuales no existen mecanismos adecuados de corrección de conflictos al interior del propio estado. Estos conflictos son generados en ocasiones porque el Presidencialismo Puro, por su excesiva rigidez, impide la solución pacífica y democrática de las crisis de poder que se den al interior del régimen 16.

Por otro lado existe el problema de la llamada Legitimidad Dual, fenómeno por el cual en las naciones con un sistema de gobierno de corte presidencial tanto el Congreso y Presidente de la República tienen la misma legitimidad democrática derivada de ser ambos órganos elegidos directamente por el pueblo. En consecuencia, en términos de democracia representativa, no existe principio constitucional alguno que resuelva un eventual conflicto ${ }^{17}$. De hecho, ambos poderes se creerían depositarios de la voluntad popular.

Ante una situación de crisis, derivada de los conflictos descritos en los párrafos precedentes, existiría un principio democrático para resolver el impasse: el traslado de la disputa al electorado, vía la disolución parlamentaria, para que el mismo la resuelva. Si el electorado considera que el Gobierno (o la mayoría parlamentaria) tienen la razón en la disputa, elegirá un Parlamento conformado en forma mayoritaria por miembros del

15 La excepción más cercana la regla señalada es el régimen peruano, el mismo que desde la Constitución de 1979 incluye la disolución parlamentaria en su articulado, no obstante que la tradición del modelo es eminentemente presidencial. Otra excepción está conformada por el régimen uruguayo, que incluye igualmente una institución equivalente.

16 Debemos precisar que la salida en Latinoamérica a las crisis ha sido, en la generalidad de los casos, el Golpe de Estado, y como resultado de este, una Dictadura, sea civil o militar. Acto seguido, la necesaria vuelta a la institucionalidad democrática producto de la imposibilidad de seguir manteniendo el Gobierno de Facto genera, normalmente, una Reforma Constitucional o la promulgación de una nueva Carta Política. El problema a este nivel radica en que dicha acción política, lejos de crear mecanismos que eviten una crisis como la anterior, busca tan solo dar una razón de ser al régimen precedente.

17 Juan Linz, "Los peligros del presidencialismo". En: Formas de Gobierno, Relaciones Ejecutivo Parlamento. Lima, Comisión Andina de Juristas, 1993, pp. 13-14. 
partido gobernante. Si no es así, la mayoría estará conformada por la oposición, con lo cual, el Gobierno deberá llegar a un acuerdo, o renunciar.

Existe otro fenómeno que justifica la existencia de una institución como la disolución parlamentaria. Si asumimos, como lo estamos haciendo hasta el momento, que a la política puede aplicarse el análisis económico, debemos notar que a fin de asegurar la calidad del producto, en todo mercado, debe asegurarse también un adecuado sistema de libre competencia. Sin embargo, la competencia del político se centra únicamente al momento de las elecciones. Esta es precisamente la principal diferencia entre la competencia a la que se ve sometido el político y la de un empresario común y corriente. La competencia en el mercado es continua. En contraposición, la competencia política es intermitente: una decisión resulta vinculante por un tiempo determinado. En el mercado patrimonial coexisten competidores que sobreviven. Uno de los principales problemas de los regímenes políticos rígidos es que la competencia en general destruye al competidor. Es jugar al todo o nada. La captura del mercado da el mercado entero a un solo oferente ${ }^{18}$.

Los norteamericanos han podido paliar las serias dificultades generadas por el efecto «todo o nada», en cuanto a la representación parlamentaria, creando elecciones cada dos años. Ahora bien, justo es reconocer que en lo concerniente al gobierno no existe mecanismo alguno que asegure la competencia a la que hemos hecho referencia en el párrafo precedente. Lo antes expresado se nota por ejemplo en el hecho de que en general, salvo algunas excepciones, los presidentes norteamericanos que vuelven a postular son reelegidos en forma inmediata ${ }^{19}$.

En el supuesto de la existencia de una institución como la disolución parlamentaria, los políticos se encontrarán en todo momento al pendien-

18 Al respecto: Juan Linz, Op. cit. p. 17. Asimismo: Buchanam y Wagner, «La Economía Keynesiana en la política democrática" En: Déficit del sector público y democracia. Ediciones Rialp S.A., Madrid, 1983.

19 Esto explica, por ejemplo, el hecho que el presidente Franklin D, Roosevelt haya sido elegido cuatro veces, y como resultado de ello, que la XXII Enmienda a la Constitución Norteamericana limitara la posibilidad de reelección a únicamente un período de gobierno adicional. Sin embargo, debemos tener en cuenta que la rigidez del sistema presidencial norteamericano y lo relativamente corto que resulta ser el periodo de gobierno del Jefe de Estado en términos del Derecho Comparado podría ayudar a corregir de alguna forma la falta de competencia política en términos de mercado y permitiría aminorar el efecto todoo nada al que hemos hecho referencia. 
te de sus acciones, puesto que al darse esta disolución se volverá a realizar elecciones. Asimismo, el gobierno, temeroso de enfrentarse con la posible competencia en el supuesto de que un parlamento adverso fuese elegido, también mejorará la calidad de su gestión ${ }^{20}$.

Asimismo, la disolución parlamentaria permite, corregir las crisis de representatividad que muestra el sistema en determinadas circunstancias. En todo el planeta, la experiencia ha demostrado que muchas veces la representatividad de la que goza un parlamento o un gobierno determinado se agota antes que el período constitucional correspondiente concluya. Si asumimos que la razón de la existencia de los sistemas de representación política consiste en «simular» la decisión a la que habría llegado el electorado en su conjunto si se pudiese poner de acuerdo ${ }^{21}$, esa justificación estaría comprometida si es que el Estado no puede ya simular la decisión respecti-

20 Es necesario señalar que para que esto tenga un resultado más eficiente, es menester que las categorías de jefe de estado y de gobierno se encuentran separadas. Ciertos autores se han pronunciado en el sentido que el sistema presidencial de inspiración norteamericana, que coloca ambas categorías sobre una sola persona (el presidente), no conjura de manera alguna las crisis políticas, sean estas derivadas de la representatividad o de la controversia entre poderes.

Asimismo, a fin de hacer posible que la disolución parlamentaria realmente funcione, deberán existir mecanismos que permitan que el Parlamento pueda revocar el mandato de ciertos miembros del gobierno, a fin de generar el contrapeso adecuado. Mecanismos como la censura ministerial o el rechazo de la cuestión de confianza y las diversas variantes de ambos que existen en el derecho comparado.

En este sentido, los sistemas parlamentarios europeos parecerían, en su diseño, ser económicamente más consistentes (y por ende, socialmente más eficientes) que los sistemas presidenciales propios del Continente Americano. Sin embargo, en los esquemas parlamentarios más puros, la estabilidad del régimen depende demasiado del control que posee el líder del partido que tiene mayoría en el Parlamento sobre éste mismo, así como de la disciplina de voto de los partidos existentes. En naciones en las cuales no funcionan los modelos más o menos puros, es necesario la puesta en práctica de esquemas más bien híbridos o mixtos, diseñados en función de cada realidad concreta.

Un desarrollo muy amplio de la antes expuesto se puede encontrar en Christian Guzmán Napurí, «Relaciones de Gobierno. Poder Ejecutivo vs Parlamento». Tesis para optar el Título de Abogado. Lima, PUCP, 1997.

21 Conforme aumenta la cantidad de ciudadanos, los costos que genera ponerse de acuerdo respecto a cuestiones de gobierno hacen inviable el modelo de democracia, pues se requerirían reuniones y votaciones para cada decisión a tomar. El sistema de sufragio universal, la creación de un Parlamento y los mecanismos legales de toma de decisiones estatales reducen tales costos. La solución legal simula el acuerdo al que llegarían los electores si pudiesen ponerse de acuerdo entre ellos. 
va. Como resultado, tendría pues que recomponerse la representación a fin que se pueda cumplir con el presupuesto antes indicado.

\section{El caso de la renovación parcial del Parlamento}

Por otro lado, es preciso indicar que otro mecanismo sumamente útil en este contexto, y que permitiría sobretodo corregir crisis de representatividad, es la elaboración de mecanismos de renovación parlamentaria por tercios o por mitades. Es decir, el permitir que cada cierto tiempo (ello depende de la duración del mandato parlamentario en cada país en concreto) el Electorado pueda modificar, por lo menos en una parte, la estructura del Parlamento, modificación que responderá necesariamente a la situación de panorama jurídico y político en el momento dado.

La renovación parcial, de alguna forma y en lo que respecta a la representación parlamentaria, palia también el efecto todo o nada - al que hemos hecho referencia en líneas anteriores - resultante de las elecciones parlamentarias. Asimismo, genera un mecanismo en el cual el elector puede corregir la composición de un Parlamento que no encuentra correspondencia con la correlación de las fuerzas políticas en la realidad política concreta. Finalmente, permite aumentar la cantidad de ciudadanos que podrían ocupar un escaño en algún momento, dada la reducción relativa del mandato parlamentario 22 .

Sin embargo, encontramos posibles circunstancias que generarían la necesidad de un diseño cuidadoso de la renovación parcial del Parlamentos. Una de ellas implica que los miembros del Parlamento gozan de un tiempo menor para informarse de las cuestiones de Estado, lo cual podría complicar la toma de decisiones. Ello ocasionaría una dependencia mayor de la administración pública y de los lobbyes. Otro elemento a tener en cuenta es la permanente cercanía de las elecciones, que acentuaría la tendencia observada, - coherente con el comportamiento racional del funcionario público - de que los parlamentarios trabajen con miras a la siguiente elección ${ }^{23}$. Para ello resulta indispensable, como lo hemos indicado líneas arriba, la creación de mecanismos que permitan conciliar la necesidad de utilidad personal del parlamentario con la eficiencia social

22 Peter Dienel y Hans Harms, Op. cit., p. 47.

23 Ibid, loc. cit. 


\subsection{Control Jurisdiccional de los llamados «Actos Políticos»}

Por otro lado, desde el punto de vista constitucional, es importante analizar la posibilidad de la existencia de mecanismos de control del Estado, por parte de los particulares, que se haga efectivo a través del uso directo de los Órganos Jurisdiccionales. En este orden de ideas, cierto sector de la doctrina, en especial en el ámbito norteamericano, señala que no resultaría posible controlar jurisdiccionalmente - y en consecuencia, jurídicamente - los llamados actos politicos, puesto que los mismos forman parte de prerrogativas que no se encuentran delimitadas y que tienen relación con la oportunidad en que las citadas decisiones se toman. Cuando la doctrina se refiere a «actos políticos» o "actos de direccionalidad política», hace alusión a aquellos actos en los cuales el Estado actúa en términos de oportunidad, teniendo plena libertad para definir que materias debieran ser consideradas de interés público.

De acuerdo a lo antes señalado, solamente podría impugnarse en sede judicial aquellas acciones basadas en facultades que se encuentran taxativamente reguladas, lo cual complicaría más de lo debido la situación generada por la posibilidad de un ejercicio ilimitado del poder por parte del Estado.

Queda claro que, por lo menos en el modelo presidencial, la limitación del accionar jurisdiccional tiene su razón de ser en la existencia del principio de separación de poderes, a través del cual se considera que el control de los actos políticos resulta ser, o debería ser, únicamente de índole política. Entonces bastaría, según el razonamiento precitado, con el control político mutuo, por más limitado que éste sea, para asegurar la calidad del accionar del Estado. Esto justifica además, como hemos señalado reiteradamente, que el sistema constitucional norteamericano sea un régimen caracterizado por una de separación de poderes rígida o tajante, con muy limitados puntos de contacto entre los órganos que conforman el Estado, lo que Karl Loewenstein denomina interdependencia por coordinación a diferencia de la interdependencia por subordinación propia de los modelos parlamentarios ${ }^{24}$. La posibilidad de una intromisión directa de la judicatura en asuntos considerados "políticos» echaría por tierra gran parte del acotado principio de separación rígida.

24 Karl Loewenstein; Op. cit., p. 132 y ss. 
Es necesario señalar que en los Estados Unidos, que es donde se elaboró la acotada teoría, existen corrientes recientes que permiten cierto matiz en la doctrina antes reseñada. Dicho matiz permite que las materias que tienen que ver directamente con el contenido de los actos políticos no puedan ser controlados, sino más bien las cuestiones de procedimiento relacionadas con dichos actos. Esto tiene sentido, en especial, en naciones en las cuales se admite que la judicatura tiene la posibilidad real de aplicar directamente la Constitución. Ello fundamentalmente para definir si la emisión de una norma administrativa o legal ha sido emitida, o su contenido está de acuerdo, con lo expresado por la norma constitucional.

Cuando existe un sistema de control difuso de constitucionalidad, o un sistema híbrido, lo señalado en el párrafo anterior es posible indubitablemente. Sin embargo, cuando el control es únicamente concentrado, la situación puede complicarse, puesto que podría interpretarse que el ordenamiento jurídico, en principio, no concede al Juez el uso de mecanismos de aplicación directa de la constitución, dado que no le concede la posibilidad de emplear control de constitucionalidad alguno respecto de las normas legales ${ }^{25}$. Es necesario precisar que, en el esquema norteamericano, esto parece compensar la excesiva rigidez del sistema y permite un mayor control de los actos políticos por parte de los particulares.

Ahora bien, la doctrina de la supuesta existencia de actos del Estado que no son susceptibles de control judicial se encuentra en franca retirada. Ello no quiere decir que no existan ciertos ordenamientos jurídicos que aun mantengan actos que no son susceptibles de análisis jurídico alguno. Sin embargo, consideraciones que hemos reseñado en párrafos anteriores, relativas a la necesidad de control del Estado por parte de los particulares, justifica plenamente la existencia del control judicial de la administración pública aun cuando ésta haga uso de facultades supuestamente discrecionales.

25 En el caso español, por ejemplo, algunos autores consideran que la existencia de un modelo concentrado de control de constitucionalidad no es óbice para que el juzgador pueda aplicar la Constitución. Sobre el particular: Eloy Espinosa Saldaña B., «Algunos alcances sobre la posibilidad de revisión judicial de aquellos actos calificados como «políticos» 0 «de direccionalidad política»». En: Revista Jurídica del Perú, Lima, Normas Legales S.A., Año XLVIII, No 14, Enero-Marzo, 1998, pp. 77-78. 


\section{Control de la Administración Pública}

Las constituciones, por lo menos desde el punto de vista del control estatal, deberían contener mecanismos a través de los cuales el administrado pueda contrarrestar los actos de la Administración Pública como tal, actos que lo perjudiquen indebidamente en forma directa. Desde el punto de vista doctrinario, se entiende que la administración pública es aquella entidad del Estado encargada del control y manejo de los servicios y bienes públicos, los mismos que se encuentran a cargo de dicho Estado.

En este orden de ideas, la Administración Pública, al gozar de atribuciones derivadas del poder del Estado (denominadas genéricamente ius imperium), puede al actuar afectar el interés del particular, el cual debería gozar de mecanismos adecuados para protegerse. La justificación la encontramos en el hecho ineludible que si bien es cierto el Derecho Administrativo implica la obligación de la administración de actuar bajo consideraciones de interés público ${ }^{26}$, también es cierto que quien toma las decisiones, llámese funcionario público, es un ser humano como cualquier otro, que decide no necesariamente en términos de interés público sino más bien, como todo ser racional, buscando maximizar el beneficio de su cargo. La principal función del derecho administrativo debería ser entonces el canalizar adecuadamente el comportamiento autointeresado del funcionario público a fin que coincida con el interés común.

Desde este punto de vista, toda constitución debe prever, aun cuando sea solamente de manera enunciativa, ciertos procesos de naturaleza judicial que permitan al particular proteger su interés. A estos procesos la doctrina las denomina genéricamente procesos de control de la administración. En principio, debería ser posible por lo menos hacer efectivos tres tipos de pretensiones: en primer lugar, el impugnar las decisiones emitidas por la autoridad administrativa, por otro lado, conseguir comprometer a la autoridad en el cumplimiento de una obligación determinada; y, finalmente, el permitir la impugnación de las normas emitidas por la

26 Existen interminables discusiones respecto a la naturaleza del concepto de interés público y al manejo de los ineludibles conflictos que se generan al enfrentarlo con el interés particular de cada individuo. Se entiende, a grandes rasgos, que interés público es el que pertenece a la generalidad de los ciudadanos y los favorece a todos. Sin embargo, para algunos el conflicto entre éste y el interés particular no tiene razón de ser, puesto que el conflicto mismo generaría que el interés público dejase de serlo. 
administración con carácter general que se opongan a la Constitución o las leyes. ${ }^{27}$

Debemos señalar que el control antes referido debe iniciarse a instancia de parte y la demanda deberá ser interpuesta ante el Poder Judicial, única entidad que podría, en uso de su autoridad, obligar a la Administración al cumplimiento de una decisión determinada. Y por si lo expuesto no fuera suficiente, un proceso judicial tiene la ventaja adicional de permitir el control directo de su tramitación por parte del particular y la obligatoria sujeción al proceso por parte de la administración. ${ }^{28}$

A este nivel, cabe hacer una acotación en cuanto a lo señalado cuando describíamos el llamado control jurisdiccional de los llamados actos políticos. En primer lugar muchos autores señalan que cuando el Estado actúa en uso de su función administrativa no cabe la existencia de actos políticos o que se consideren intrínsecamente discrecionales, siendo incluso las resoluciones y normas respecto de los cuales hay un margen amplio de acción, actos susceptibles de ser impugnados judicialmente ${ }^{29}$.

En segundo lugar, es necesario señalar que cuando nos referimos a actividad discrecional hacemos referencia a aquella que no depende de normas legislativas concretas y preexistentes que la regulen. Las facultades discrecionales le permiten al órgano administrativo cierta libertad para elegir entre uno u otro curso de acción, o para hacer una cosa de una u otra forma. La necesidad de dejar a la Administración Pública cierta actividad discrecional deriva, en principio, de la circunstancia de que es imposible para el legislador tener una visión exacta de los elementos concretos que, al menos en parte y en ciertos casos, condicionan la oportunidad de la decisión administrativa. Ahora bien, la actividad discrecional en realidad no es tal, pues se encuentra limitada por el ordenamiento jurídico, y en especial, por los derechos fundamentales.

27 En el ámbito nacional, dichos procesos se denominan, respectivamente, acción contencioso administrativa, acción de cumplimiento y acción popular.

28 Es necesario señalar, sin embargo, que el funcionamiento de estos mecanismos de control depende en gran medida de la independencia que en el texto constitucional y en los hechos se garantiza al Organo Judicial en cada nación en concreto.

29 En el derecho comparado, sin embargo, existen ciertas resoluciones administrativas que no son susceptibles de impugnación judicial por mandato del propio texto constitucional. En el caso peruano, existen dos específicos: Las resoluciones emitidas por el Jurado Nacional de Elecciones y las emitidas por el Consejo Nacional de la Magistratura. 


\section{Los costos generados por el control elector-Estado}

Un tema fundamental a tomar en cuenta para que sea posible el traslado de ciertas decisiones políticas al electorado es el de determinar cuales son los costos en los que incurriría, por un lado el Estado (costos administrativos o de organización), y por otro lado los particulares (costos de transacción $)^{30}$, teniendo en cuenta que el costo del Estado es asumido por los particulares a través del sistema tributario.

En este orden de ideas es necesario señalar que los sistemas de participación del electorado en las decisiones públicas se justifican siempre y cuando los costos resultantes de efectuar el correspondiente traslado sean menores que los costos en que se incurriría si la decisión fuese tomada por la autoridad política. El umbral que existe entre que decisiones políticas son tomadas por el Estado y cuales se trasladan, o debieran trasladarse, al electorado, se define entonces en términos de utilidad inmediata.

\section{El Teorema de Coase}

El llamado Teorema de Coase es muy útil para entender como definir el umbral que existe entre aquellas decisiones de la autoridad de deben trasladarse al electorado y aquellas decisiones que debe tomarse sin intervención de la ciudadanía. Por un lado, porque permite al legislador discriminar entre hacer que el Estado asuma el costo social de sus decisiones o crear las condiciones que permitan que éste sea asumido por los particulares - en este caso, el electorado - con el menor costo posible. Por otro lado, sin embargo, el Teorema de Coase nos permite justificar, desde el punto de vista del mercado político, la asignación de derechos de participación mediante el uso de las propias normas constitucionales y legales a fin de corregir las dificultades que tiene dicho mercado para garantizar plenamente el ejercicio de las libertades públicas por parte del elector.

El Teorema de Coase se enuncia entonces de la siguiente manera: «si los costos de transacción son sensiblemente iguales a cero, no importa la solución legal que se adopte, pues siempre las partes involucradas, a través de transacciones en el mercado, llegarán a la solución más eficiente». ${ }^{31}$

30 Llamamos costo de transacción al costo que genera el que los particulares lleguen a acuerdos respecto a cuestiones de importancia.

31 Alfredo Bullard, «Ronald Coase y el Sistema Jurídico. Sobre el Nobel de Economía 1991\%. En Apuntes No 28, 1991. Michell Polinsky, An Introduction to Law and 
Es decir, únicamente cuando los costos son muy elevados se justifica la existencia de una solución legal que permita la eficiencia social. Por ello, la intervención estatal debe restringirse únicamente a la asignación de derechos a través de la norma legal. En este orden de ideas, y considerando que el concepto de costo social debe entenderse como el más amplio posible es necesario un análisis cuidadoso a fin de determinar si el costo administrativo de tomar decisiones políticas, comparado con los costos de transacción que tengan que asumir los particulares justifica o no el traslado de las citadas decisiones.

Siguiendo con el mismo razonamiento, y de acuerdo al Teorema de Coase, si los costos de transacción tienden a cero no interesa la solución legal adoptada, pues siempre se llegará a la solución más eficiente. Por otro lado, si los costos son altos, será necesario implementar una solución legal que los conjure los mismos. Como indicáramos líneas arriba, la razón de ser de la democracia representativa estriba en que el costo de que los particulares tomen por sí mismos todas las decisiones políticas resulta prohibitivo, razón por la cual es necesario que una parte importante de estas decisiones sean tomadas por sus representantes, elegidos para el efecto.

Cabe precisar que, desde el punto de vista constitucional, el costo social se encuentra cifrado también en conceptos como gobernabilidad, Estado de Derecho, estabilidad política y otros más que podemos encontrar en las constituciones de naciones democráticas. En consecuencia, el costo de la toma de decisiones implica la necesidad de tomar en cuenta dichos conceptos al momento de realizar la correspondiente evaluación.

Asimismo, los costos de ponerse de acuerdo impiden, en general, la elaboración de reglas de toma de decisiones distintas a las que se derivan del voto directo, en las cuales la decisión se toma en forma inmediata puesto que las alternativas de elección se encuentran predeterminadas. El voto directo, sumado a la ausencia de debate, reduce plausiblemente los costos de transacción en los que incurriría el electorado para tomar la decisión política respectiva.

Por otro lado, no todas las decisiones políticas pueden trasladarse. En un primer término, es poco probable que el Estado inicie algún proceso de participación política por propia iniciativa, sino más bien a través del accionar del propio electorado mediante los mecanismos que la Ley esta- 
blece. Ello, a menos que la propia Constitución obligue a la iniciación del proceso ante un supuesto determinado. Esto último reduciría los costos en los que incurre el electorado al tomar la iniciativa para activar los mecanismos establecidos.

En segundo término, las constituciones establecen ciertas limitaciones para aquellos temas que pueden resultar siendo materia de decisión del electorado. Ello, fundamentalmente por el temor a que la opinión pública pueda ser manipulada de alguna forma por el propio Estado u otros entes interesados en el resultado del proceso.

\section{El Problema de los Costos Administrativos}

Los costos administrativos elevados pueden generar dificultades para elaborar mecanismos adecuados de participación política. Los costos que son asumidos el Estado consisten principalmente en el gasto realizado al organizar el proceso a través del cual se hace efectivo el mecanismo de participación. En el caso, por ejemplo, de la iniciativa legislativa, no hay problemas mayores puesto que quien realiza el proceso es el Parlamento, el cual tramita el proyecto de ley ciudadano como cualquier otro proyecto sometido a su consideración por parte de cualquiera de los entes o personas legitimadas para presentarlo. Sin embargo, si nos referimos a los mecanismos que requieren del voto directo de los ciudadanos, la organización es prácticamente la misma que la de un proceso electoral, con el consiguiente gasto en términos presupuestarios.

Por otro lado, el tema de los costos administrativos adquiere especial relevancia cuando notamos que el Estado es financiado principalmente a través del sistema impositivo, el mismo que se encuentra dirigido hacia la sociedad en su conjunto. En la práctica, el propio electorado solventa los mecanismos de participación a través de la canalización que realiza el Estado de los recursos que recibe.

Finalmente, el análisis a fin de determinar la conveniencia del traslado de decisiones políticas es implica conceptos de diversa naturaleza, en términos de costo y beneficio, en los cuales debe tomarse en cuenta factores que serían distintos entre los mismos. Inicialmente, y por lo menos en el nivel de costos administrativos, pareciera que el análisis involucra comparar conceptos políticos con conceptos meramente patrimoniales. En cierta medida, la Teoría de la Elección Pública nos muestra que en la realidad se va a dar la necesidad de la comparación antes citada. En suma, las deci- 
siones que se toma debe maximizar la utilidad del resultado, con el menor costo social posible, en el sentido más amplio del término.

\section{Conclusiones finales}

Como resultado del análisis precedente, debemos concluir que el análisis económico es consistente con la existencia de los mecanismos de control estatal por parte de los particulares. Como resultado de ello, se concluye que no basta el principio de separación de poderes (o el de control entre órganos del Estado) para asegurar la libertad política. Deberá ser posible, en determinadas circunstancias y bajo ciertas condiciones, trasladar las decisiones políticas o el control de éstas al electorado. Para ello se han establecido mecanismos de participación política, mecanismos de modificación de la estructura del gobierno a través del traslado de disputas del Estado al electorado y mecanismos de control de actos del Estado y la Administración Pública.

Sin embargo el límite de los traslados anteriormente citados deberá estar determinado, en primer lugar, por los costos de transacción que debe asumir el electorado a fin de tomar la decisión política determinada. Asimismo debemos tener en cuenta los costos administrativos que puedan generar los mecanismos puestos en práctica constitucionalmente, teniendo en cuenta el hecho que los costos son asumidos por el propio electorado a través del sistema impositivo. 